\title{
Resultados Hospitalares da Intervenção Coronária Percutânea Primária no Infarto Agudo do Miocárdio em Pacientes com Mais de 80 Anos
}

\author{
Nelson Ricardo Thomas Júnior ${ }^{1}$, Marcelo J. C. Cantarelli', Helio J. Castello Junior' ${ }^{1}$, Silvio Gioppato', \\ Rosaly Gonçalves' ${ }^{1}$, João Batista F. Guimarães ${ }^{1}$, Patricia Teixeira da Silva' ${ }^{1}$, Higo Cunha Noronha', \\ Leonardo Cao C. Oliveira ${ }^{1}$, Evandro K. P. Ribeiro ${ }^{1}$
}

\section{RESUMO}

Introdução: $\mathrm{O}$ aumento da expectativa de vida da população e o risco de sangramento com o uso de trombolíticos têm permitido que pacientes cada vez mais idosos com infarto agudo do miocárdio sejam tratados pela intervenção coronária percutânea (ICP) primária. Analisamos os resultados hospitalares da ICP primária em pacientes com idade $\geq 80$ anos. Método: No período de janeiro de 2002 a outubro de 2008, foram realizadas 4.788 ICPs, sendo 428 ICPs primárias. Destas, 34 foram em pacientes com idade $\geq 80$ anos e 394 em pacientes $<80$ anos. Resultados: $O$ sexo feminino $(47,1 \%$ vs. $27,7 \%$; $P=0,017)$, a classe funcional Killip IV $(11,8 \%$ vs. $4,1 \%$; $P=0,002)$ e o fluxo coronário TIMI $2 / 3$ pré-ICP $(60 \%$ vs. $44,4 \% ; P=0,032)$ foram mais frequentes nos idosos. Tabagismo $(44,9 \%$ vs. $8 \%$; $\mathrm{P}<0,001)$ e uso de inibidores da glicoproteína IIb/IIla $(32,5 \%$ vs. $11,7 \% ; P=0,04)$ prevaleceram no grupo $<80$ anos. Não ocorreram diferenças significativas quanto ao tempo portabalão $(117,4 \pm 125,7$ minutos vs. $179,3 \pm 169,8$ minutos; $\mathrm{P}=0,095)$, à taxa de utilização de stents $(91,2 \%$ vs. $98,2 \%$; $P=0,78)$, e ao uso de stents farmacológicos (0 vs. 2,8\%; $\mathrm{P}=0,067)$ ou de dispositivos de aspiração de trombos $(11,7 \%$ vs. 12,$2 ; P=0,65)$. Não houve diferença quanto à taxa de sucesso angiográfico (97,1\% vs. 96,5\%; P > 0,99), porém o grupo $\geq 80$ anos apresentou mortalidade hospitalar mais elevada $(11,8 \%$ vs. $2,3 \% ; P=0,014)$. Reinfarto, acidente vascular cerebral, complicações vasculares maiores e insuficiência renal aguda foram semelhantes entre os grupos. Conclusão: A ICP apresenta, em geral, altas taxas de sucesso angiográfico e baixo índice de complicações, reforçando seu papel como método preferencial de reperfusão na fase aguda do infarto do miocárdio. Os pacientes muitos idosos, com mais de 80 anos, apresentam maior mortalidade, possivelmente reflexo da maior comorbidade desse grupo.

DESCRITORES: Angioplastia transluminal percutânea coronária. Idoso de 80 anos ou mais. Infarto do miocárdio.
ABSTRACT

In-hospital Results of Primary Percutaneous Coronary Intervention in Patients Above 80 Years of Age

Background: Improvements in life expectancy of the overall population and the risk of bleeding with thrombolytics have enabled very elderly patients with acute myocardial infarction to be treated by primary percutaneous coronary intervention $(\mathrm{PCl})$. We analyzed the primary $\mathrm{PCl}$ in-hospital results in patients $\geq 80$ years of age. Method: From January 2002 to October 2008, 4,788 PCls were performed, of which 428 were primary $\mathrm{PCl}$. Of these, 34 were performed in patients $\geq 80$ years of age and 394 in patients $<80$ years of age. Results: Females $(47.1 \%$ vs. $27.7 \% ; \mathrm{P}=0.017)$, Killip IV functional class $(11.8 \%$ vs. $4.1 \% ; \mathrm{P}=0.002)$ and pre-procedure TIMI $2 / 3$ flow $(60 \%$ vs. $44.4 \% ; \mathrm{P}=0.032)$ were more frequent in elderly patients. Smoking $(44.9 \%$ vs. $8 \%$; $\mathrm{P}<0.001)$ and use of glycoprotein Ilb/IIla inhibitors $(32.5 \%$ vs. $11.7 \% ; P=0.04)$ were prevalent in the group $<80$ years of age. There were no significant differences for door-to-balloon time $(117.4 \pm 125.7$ minutes vs. $179.3 \pm$ 169.8 minutes; $P=0.095)$, use of stents $(91.2 \%$ vs. $98.2 \%$; $\mathrm{P}=0.78)$, or drug-eluting stents (0 vs. $2.8 \% ; \mathrm{P}=0.067)$ and thrombus aspiration devices $(11.7 \%$ vs. $12.2 \%$; $\mathrm{P}=0.65)$. Angiographic success rate was equal between the groups $(97.1 \%$ vs. $96.5 \%$; $\mathrm{P}>0.99)$, but patients $\geq 80$ years old had a higher mortality rate $(11.8 \%$ vs. $2.3 \%$; $P=0.014)$. Reinfarction, stroke, major vascular complications and acute renal failure were similar between groups. Conclusion: $\mathrm{PCl}$ usually presents high angiographic success and low complication rates, reinforcing its role as a method of choice for reperfusion in the acute phase of myocardial infarction. Very elderly patients ( $\geq 80$ years of age) have higher mortality rates, possibly due to the greater comorbidity in this group.

KEY-WORDS: Angioplasty, transluminal, percutaneous coronary. Aged, 80 and over. Myocardial infarction.

1 Hospital Bandeirantes - São Paulo, SP, Brasil.

Correspondência: Nelson Ricardo Thomas Júnior. Rua Galvão Bueno, 257 - Liberdade - São Paulo, SP, Brasil - CEP 01506-000

E-mail: nelsonthomasjr@gmail.com

Recebido em: 8/12/2009 - Aceito em: 16/2/2010 
O s pacientes idosos, com idade acima dos 65 anos, constituem uma população responsável por $85 \%$ das mortes por infarto agudo do miocárdio. O envelhecimento do sistema cardiovascular e as comorbidades associadas à senilidade limitam a reserva cardíaca e aumentam as complicações relacionadas ao tratamento da injúria miocárdica aguda..$^{1-3}$

O aumento da expectativa e da qualidade de vida da nossa população e o risco de sangramento com o uso de fibrinolíticos têm levado indivíduos cada vez mais idosos, com quadro de infarto agudo do miocárdio, a serem tratados por meio da intervenção coronária percutânea primária. ${ }^{4-6}$ No entanto, em pacientes idosos, a intervenção coronária percutânea tem mostrado maior risco inerente ao procedimento, em comparação aos mais jovens. ${ }^{7}$ Entre os mais idosos, com idade acima dos 80 anos, estudo recente sugere não haver superioridade dos resultados clínicos da intervenção coronária percutânea em relação à trombólise, demonstrando que essa população ainda representa um desafio ao tratamento. ${ }^{8}$

Em nosso meio, poucos trabalhos têm avaliado os resultados clínicos da intervenção coronária percutânea primária em pacientes muito idosos (acima dos 80 anos de idade). Dessa forma, buscamos neste estudo avaliar os resultados hospitalares da intervenção coronária percutânea primária nessa população.

\section{MÉTODO}

\section{Pacientes}

No período de janeiro de 2002 a outubro de 2008, foram realizadas 4.788 intervenções coronárias percutâneas em nosso serviço, sendo 428 (8,9\%) primárias.

Foram incluídos nesse estudo todos os 428 pacientes submetidos a intervenção coronária percutânea primária, sendo divididos para a análise em dois grupos: $\geq 80$ anos $(\mathrm{n}=34)$ e $<80$ anos $(\mathrm{n}=394)$.

Analisamos as características clínicas e angiográficas relacionadas ao procedimento, as taxas de sucesso e a evolução clínica até a alta hospitalar.

Os dados foram coletados prospectivamente e armazenados em um banco de dados informatizado.

\section{Intervenção coronária percutânea}

As intervenções foram realizadas em quase sua totalidade por via femoral, excetuando-se um paciente do grupo $<80$ anos em que foi utilizada a via radial. A técnica e a escolha do material durante o procedimento ficou a cargo dos operadores, assim como a necessidade do uso de inibidores da glicoproteína IIb/IIla. Foi utilizada heparina não-fracionada no início do procedimento, na dose de $70 \mathrm{U} / \mathrm{kg}$ a $100 \mathrm{U} / \mathrm{kg}$, exceto em 6 pacientes do grupo $<80$ anos que já haviam recebido heparina de baixo peso molecular nas unidades de emergência. Todos os pacientes receberam terapia antiplaquetária combinada com ácido acetilsalicílico na dose de ataque de $300 \mathrm{mg}$ e manutenção de $100 \mathrm{mg} / \mathrm{dia}$ a $200 \mathrm{mg} / \mathrm{dia}$, e clopidogrel na dose de ataque de $300 \mathrm{mg}$ a $600 \mathrm{mg}$ e manutenção de $75 \mathrm{mg} / \mathrm{dia}$ ou ticlopidina $250 \mathrm{mg}$ a cada 12 horas.

\section{Análise angiográfica e definições}

As análises foram realizadas em pelo menos duas projeções ortogonais por operadores experientes com uso de angiografia quantitativa digital. Utilizamos neste estudo os mesmos critérios angiográficos constantes no banco de dados da Central de Intervenções Cardiovasculares (CENIC) da Sociedade Brasileira de Hemodinâmica e Cardiologia Intervencionista. O tipo de lesão foi classificado conforme os critérios do American College of Cardiology/American Heart Association. ${ }^{9}$ Foram consideradas lesões longas aquelas com comprimento superior a $20 \mathrm{~mm}$. Para a determinação do fluxo coronário pré e pós-procedimento foi utilizada a classificação de TIMI. ${ }^{10}$ Sucesso angiográfico foi definido como redução do diâmetro de estenose da lesão-alvo $<30 \%$, restabelecimento e manutenção do fluxo anterógrado normal (grau TIMI 3). ${ }^{11} \mathrm{O}$ reinfarto foi definido pela presença de alterações eletrocardiográficas (novo supradesnivelamento do segmento ST ou novas ondas Q) e/ou pela evidência angiográfica de oclusão do vasoalvo. Foi considerada cirurgia de revascularização de emergência aquela realizada imediatamente após a intervenção coronária percutânea. Foram consideradas para análise a mortalidade geral (óbito por qualquer causa) e a mortalidade cardíaca (choque cardiogênico, insuficiência cardíaca, reinfarto, ruptura cardíaca, arritmia e morte súbita) no período hospitalar. Foram agrupadas como complicações vasculares maiores: hematoma $>10 \mathrm{~cm}$ no local da punção arterial, sangramento com queda de hemoglobina $>3 \mathrm{~g} / \mathrm{dl}$ ou necessidade de transfusão sanguínea, e ocorrência de pseudoaneurisma, hematoma retroperitoneal ou fístula arteriovenosa com necessidade de correção cirúrgica. Insuficiência renal aguda foi definida como elevação de $0,5 \mathrm{mg} / \mathrm{dl}$ no valor absoluto da creatinina sérica 48 a 72 horas após a intervenção coronária percutânea.

\section{Análise estatística}

Os dados armazenados em banco de dados com base Oracle foram plotados em planilhas em Excel e analisados em programa estatístico NCSS versão 2004. As variáveis contínuas foram expressas em média \pm desvio padrão e as variáveis categóricas, em percentis. As associações entre as variáveis contínuas foram avaliadas pelo teste $t$ de Student. As associações entre as variáveis categóricas foram avaliadas pelos testes de qui-quadrado ou exato de Fischer nas tabelas de dimensão $2 \times 2$. Nas tabelas de maior dimensão, foram aplicados os testes de qui-quadrado ou da razão de verossimilhança $(G)$. Foi adotado nível de significância de $\mathrm{P}<0,05$. Intervalos de confiança de $95 \%$ foram 
Thomas Júnior NR, et al. Resultados Hospitalares da Intervenção Coronária Percutânea Primária no Infarto Agudo do Miocárdio em Pacientes com Mais de 80 Anos. Rev Bras Cardiol Invasiva. 2010;18(1):17-23.

representados para as variáveis contínuas quando os níveis descritivos de $\mathrm{P}$ foram significantes.

\section{RESULTADOS}

As características clínicas dos dois grupos estão expostas na Tabela 1. Em relação ao grupo $<80$ anos, houve, proporcionalmente, maior número de pacientes do sexo feminino no grupo $\geq 80$ anos $[47,1 \%$ vs. $27,7 \%$; intervalo de confiança de $95 \%$ (IC 95\%): 0,004$0,029 ; P=0,017]$, enquanto o tabagismo prevaleceu no grupo $<80$ anos $(44,9 \%$ vs. $8 \%$; IC 95\%: 0,007$0,012 ; \mathrm{P}<0,001)$. No grupo $\geq 80$ anos, ocorreu maior número de pacientes em grupo funcional avançado Killip IV (11,8\% vs. 4,1\%; IC 95\%: 0,002-0,008; P = 0,002). Não ocorreram diferenças significativas entre os dois grupos quanto à presença de hipertensão arterial, diabetes melito, dislipidemia, história de infarto agudo do miocárdio e revascularização miocárdica ou intervenção coronária percutânea prévias.

A Tabela 2 apresenta as características angiográficas. Os idosos do grupo $\geq 80$ anos apresentaram maior ocorrência de fluxo TIMI 2/3 pré-procedimento na artéria relacionada ao infarto (60\% vs. 44,4\%; IC 95\%: 0,015-0,048; $P=0,032)$. Não ocorreram diferenças significativas em relação ao acometimento multiarterial, à presença de circulação colateral, à complexidade das lesões coronárias e ao vaso-alvo.

Em relação às características dos procedimentos (Tabela 3), observou-se maior utilização de inibidores da glicoproteína IIb/IIla no grupo $<80$ anos $(32,5 \%$ vs. 11,7\%; IC 95\%: 0,021-0,058; P = 0,04), bem como a utilização de stents de maior diâmetro $(3,2 \pm 0,5 \mathrm{~mm}$ vs. $3 \pm 0,4 \mathrm{~mm} ; P=0,031)$. Não ocorreram diferenças sig- nificativas quanto ao tempo porta-balão $(117,4 \pm 125,7$ minutos vs. $179,3 \pm 169,8$ minutos; $P=0,095)$, à taxa de utilização de stents $(91,17 \%$ vs. $98,22 \%$; $P=0,79)$, ao uso de stents farmacológicos ( 0 vs. $2,8 \%$; $P=0,067$ ) ou dispositivos de aspiração de trombos $(11,7 \%$ vs. $12,2 \% ; P=0,65)$. A quantificação angiográfica das obstruções pré- e pós-procedimento também não diferiu entre os grupos.

Quanto aos resultados hospitalares da intervenção coronária percutânea primária (Tabela 4), o sucesso angiográfico foi elevado e não diferiu entre os grupos $(97,1 \%$ vs. $96,5 \%$; $P>0,99)$, porém o grupo $\geq 80$ anos apresentou mortalidade significativamente mais elevada que o grupo < 80 anos (11,8\% vs. $2,3 \%$; IC $95 \%$ : 0,002-0,025; $P=0,014)$. Todos os óbitos foram de causa cardíaca. No grupo $\geq 80$ anos, foram registrados 3 óbitos em pacientes que se apresentavam em grupo funcional Killip IV pré-intervenção coronária percutânea, decorrentes da evolução em choque cardiogênico, a despeito do sucesso angiográfico, tendo como vasosalvo a artéria descendente anterior em dois casos e o tronco de coronária esquerda em um caso. O outro óbito ocorreu durante o procedimento com insucesso na recanalização da artéria coronária direita em paciente multiarterial. Casos de reinfarto ocorreram apenas no grupo < 80 anos (0 vs. $1 \%$; P > 0,99), dentro das primeiras 24 horas pós-intervenção coronária percutânea, e foram diagnosticados por retorno da dor anginosa e reelevação do segmento ST ao eletrocardiograma nos quatro pacientes. Todos foram submetidos a nova intervenção com sucesso. Não ocorreram casos de cirurgia de revascularização de emergência em ambos os grupos. A ocorrência de acidente vascular cerebral $(2,9 \%$ vs. $0,3 \% ; P=0,067)$, de com-

TABELA 1

Características clínicas dos grupos

\begin{tabular}{|c|c|c|c|}
\hline Característica & $\begin{array}{c}\geq 80 \text { anos } \\
(n=34)\end{array}$ & $\begin{array}{c}<80 \text { anos } \\
(\mathrm{n}=394)\end{array}$ & $\mathbf{P}$ \\
\hline Idade (média \pm desvio padrão) & $83,2 \pm 2,9$ & $59 \pm 11,2$ & \\
\hline Mínima & 80 & 28 & \\
\hline Máxima & 93 & 79 & \\
\hline Sexo feminino, $\mathrm{n}(\%)$ & $16(47,1)$ & $109(27,7)$ & 0,017 \\
\hline Diabetes melito, n (\%) & $6(24)$ & $71(27,7)$ & 0,69 \\
\hline Hipertensão arterial, n (\%) & $21(84)$ & $175(68,4)$ & 0,10 \\
\hline Tabagismo, n (\%) & $2(8)$ & $115(44,9)$ & $<0,001$ \\
\hline Dislipidemia, n (\%) & $6(24)$ & $94(36,7)$ & 0,21 \\
\hline RM prévia, n (\%) & $3(8,8)$ & $17(4,3)$ & 0,21 \\
\hline ICP prévia, n (\%) & $1(2,9)$ & $18(4,6)$ & 0,24 \\
\hline IAM prévio, n (\%) & $3(12)$ & $28(10,9)$ & 0,75 \\
\hline Killip IV, n (\%) & $4(11,8)$ & $16(4,1)$ & 0,002 \\
\hline
\end{tabular}

IAM = infarto agudo do miocárdio; ICP = intervenção coronária percutânea; $\mathrm{n}$ = número de pacientes; RM = cirurgia de revascularização miocárdica. 
TABELA 2

Características angiográficas dos grupos

\begin{tabular}{lccc}
\hline Característica & $\begin{array}{c}\geq \mathbf{8 0} \text { anos } \\
(\mathbf{n}=\mathbf{3 4})\end{array}$ & $\begin{array}{c}<\mathbf{8 0} \text { anos } \\
(\mathbf{n}=\mathbf{3 9 4})\end{array}$ & $\mathbf{P}$ \\
\hline Multiarterial, $\mathrm{n}(\%)$ & $7(20,6)$ & $48(12,2)$ & 0,29 \\
Vaso-alvo, $\mathrm{n}(\%)$ & & 0,08 \\
$\mathrm{DA}$ & $15(44,1)$ & $230(52,3)$ & \\
$\mathrm{CX}$ & $6(17,1)$ & $55(12,5)$ & \\
$\mathrm{CD}$ & $12(34,3)$ & $145(32,9)$ & \\
Enxertos & $1(2,8)$ & $8(1,8)$ & 0,88 \\
TCE & $1(2,9)$ & $2(0,4)$ & 0,10 \\
Lesões tipo B2/C, $\mathrm{n}(\%)$ & $29(82,9)$ & $351(79,8)$ & 0,51 \\
Lesões longas, $\mathrm{n}(\%)$ & $103(23,4)$ & 0,032 \\
Bifurcações, $\mathrm{n}(\%)$ & $3(11,4)$ & $36(8,2)$ & 0,67 \\
Fluxo TIMI 2/3, $\mathrm{n}(\%)$ & $1(2,9)$ & $45(44,4)$ & \\
Circulação colateral, $\mathrm{n}(\%)$ & $21(60)$ & $4(11,4)$ & \\
\hline
\end{tabular}

$\mathrm{CD}=$ artéria coronária direita; $\mathrm{CX}=$ artéria circunflexa; $\mathrm{DA}=$ artéria descendente anterior; $\mathrm{n}=$ número de pacientes; $\mathrm{TCE}=$ tronco de coronária esquerda.

TABELA 3

Características dos procedimentos

\begin{tabular}{lccc}
\hline Característica & $\begin{array}{c}\mathbf{8 0} \text { anos } \\
(\mathbf{n}=\mathbf{3 4})\end{array}$ & $\begin{array}{c}\mathbf{8 0} \text { anos } \\
(\mathbf{n}=\mathbf{3 9 4})\end{array}$ & $\mathbf{P}$ \\
\hline Tempo porta-balão, minutos & $117,4 \pm 125,7$ & $179,3 \pm 169,8$ & 0,095 \\
Uso de stent, \% & 91,17 & 98,22 & 0,79 \\
$\quad$ Stents convencionais, n (\%) & $31(100)$ & $380(98,2)$ & 0,97 \\
Stents farmacológicos, n (\%) & $0(0)$ & $7(2,8)$ & 0,067 \\
Inibidores da glicoproteína Ilb/IIla, n (\%) & $4(11,7)$ & $128(32,5)$ & 0,04 \\
Tromboaspiração, n (\%) & $4(11,7)$ & $48(12,2)$ & 0,65 \\
Diâmetro dos stents, mm & $3 \pm 0,4$ & $3,2 \pm 0,5$ & 0,031 \\
Comprimento dos stents, mm & $19,7 \pm 6,6$ & $18,7 \pm 6,3$ & 0,40 \\
Grau de estenose pré, \% & $92,3 \pm 14,7$ & $94,1 \pm 10,9$ & 0,62 \\
Grau de estenose pós, \% & $9,3 \pm 16,2$ & $8 \pm 11,4$ & 0,75 \\
\hline $\mathrm{n}=$ número de pacientes. & & & \\
\hline
\end{tabular}

plicações vasculares maiores $(8,8 \%$ vs. $5,6 \% ; P=0,16)$ e de insuficiência renal aguda $(2,9 \%$ vs. $0,5 \%$; $P=0,10)$ foi semelhante entre os grupos.

\section{DISCUSSÃo}

Aproximadamente $30 \%$ de todos os pacientes com infarto agudo do miocárdio têm idade superior a 75 anos; no entanto, esses idosos nunca estiveram bem representados nos estudos clínicos clássicos de infarto agudo do miocárdio, por serem excluídos da análise ou por aparecerem em pequeno número. ${ }^{7}$ Estudos randomizados realizados a partir da última década e a adoção da intervenção coronária percutânea primá- ria como tratamento preferencial do infarto agudo do miocárdio (recomendação classe I, nível de evidência A nas principais diretrizes) respaldam hoje sua utilização em pacientes idosos..$^{8,11-14}$

O aumento do afluxo de pacientes idosos para a intervenção coronária percutânea ficou evidente em um estudo de 10 anos, com elevação temporal da proporção de pacientes $\geq 80$ anos de 4/113 (3,5\%) em 1997 para 51/579 $(8,8 \%)$ em $2007(\mathrm{P}<0,01) .{ }^{15} \mathrm{Em}$ nosso meio, Tavares Filho et al. ${ }^{16}$ demonstraram aumento da proporção de octogenários nas intervenções coronárias percutâneas realizadas no biênio 2006-2007 em comparação com o período de 2002-2003 (4,7\% vs. 3,7\%; 
Thomas Júnior NR, et al. Resultados Hospitalares da Intervenção Coronária Percutânea Primária no Infarto Agudo do Miocárdio em Pacientes com Mais de 80 Anos. Rev Bras Cardiol Invasiva. 2010;18(1):17-23.

TABELA 4

Resultados hospitalares da intervenção coronária percutânea primária

\begin{tabular}{lccc}
\hline Resultado & $\begin{array}{c}\geq \mathbf{8 0} \text { anos } \\
(\mathbf{n}=\mathbf{3 4})\end{array}$ & $\begin{array}{c}<\mathbf{8 0} \text { anos } \\
(\mathbf{n}=\mathbf{3 9 4})\end{array}$ & $\mathbf{P}$ \\
\hline Sucesso angiográfico, n (\%) & $33(97,1)$ & $380(96,5)$ & $>0,99$ \\
Mortalidade geral, n (\%) & $4(11,8)$ & $9(2,3)$ & 0,014 \\
Reinfarto, n (\%) & $0(0)$ & $4(1)$ & $>0,99$ \\
RM de emergência, n (\%) & $0(0)$ & $0(0)$ & - \\
AVC, n (\%) & $1(2,9)$ & $1(0,3)$ & 0,067 \\
Complicações vasculares & & $22(5,6)$ & 0,16 \\
maiores, n (\%) & $3(8,8)$ & $2(0,5)$ & 0,10 \\
IRA, n (\%) & $1(2,9)$ & &
\end{tabular}

AVC = acidente vascular cerebral; IRA = insuficiência renal aguda; $\mathrm{n}=$ número de pacientes; $\mathrm{RM}$ = cirurgia de revascularização miocárdica.

$\mathrm{P}=0,07)$, bem como crescente complexidade no perfil clínico/angiográfico desses pacientes.

A complexidade tanto clínica como angiográfica da população idosa eleva os riscos do procedimento e tem sido caracterizada em grande parte por maior acometimento de doenças sistêmicas graves (p. ex.: pneumopatias crônicas, insuficiência renal, insuficiência vascular periférica), maior número de revascularizações cirúrgicas e infartos prévios, e maior incidência de doença multiarterial, de lesões coronárias complexas e de fração de ejeção do ventrículo esquerdo reduzida. ${ }^{2,11}$ Em nossa casuística, não ocorreram diferenças entre os grupos quanto à presença de revascularizações cirúrgicas ou percutâneas prévias, bem como em relação à extensão e complexidade das lesões coronárias. Assim como em outros estudos, encontramos maior proporção de mulheres e taxa mais alta de fluxo coronário TIMI 2/3 pré-intervenção coronária percutânea. ${ }^{17,18}$

Pelo fato de grande parte dos pacientes de ambos os grupos não ter realizado ventriculografia esquerda (76,5\% do grupo $\geq 80$ anos e $52,5 \%$ do grupo $<80$ anos), por não ser rotina na intervenção coronária percutânea primária em nosso serviço, não procedemos à análise da função ventricular. Consideramos apenas o estado clínico funcional pela classificação Killip, sendo encontrada no grupo $\geq 80$ anos maior ocorrência da classe mais avançada (IV), evoluindo a maioria destes a óbito em choque cardiogênico. A classe Killip IV é um forte preditor de mortalidade, ao lado de idade > 75 anos, baixa fração de ejeção, baixo fluxo TIMI final, necessidade de balão intraaórtico, acidente vascular cerebral e arritmia significante, como atesta a análise conjunta dos estudos PAMI. ${ }^{17}$ Assim como na população geral, a apresentação em choque cardiogênico contribui para maior taxa de mortalidade hospitalar entre os octogenários submetidos a intervenção coronária percutânea primária. ${ }^{19} \mathrm{O}$ registro de Melbourne, ${ }^{20}$ que incluiu 4.360 pacientes, dos quais $11,3 \%(n=491)$ com idade acima de 80 anos, coloca como principais preditores independentes de mortalidade em 12 meses choque cardiogênico, idade acima de 80 anos e insuficiência renal. A apresentação clínica em infarto agudo do miocárdio para intervenção coronária percutânea foi, por si só, considerada preditor de óbito no estudo de Lemos et al., ${ }^{18}$ o que diferencia a complexidade da intervenção coronária percutânea no infarto agudo do miocárdio em relação às outras apresentações clínicas.

A taxa de utilização de stents foi elevada em ambos os grupos de nosso estudo, sendo condizente com a evolução tecnológica do procedimento. No entanto, o emprego de stents farmacológicos foi baixo e a justificativa desse fato encontra-se embasada mais em razões econômicas (limitação a seu uso por fontes financiadoras da saúde pública e suplementar) que em evidências clinicas atuais. A utilização de inibidores da glicoproteína IIb/IIla foi menor no grupo $\geq 80$ anos e talvez o receio da ocorrência de sangramentos justifique esse fato, já que sua indicação ficou a critério dos operadores. No grupo $<80$ anos, seu uso (32,5\%) esteve dentro das taxas relatadas pelo registro da Sociedade Latino-Americana de Cardiologia Intervencionista (SOLACI) no período de 2000 a 2008 (22,7\% a $40 \%) .{ }^{14} \mathrm{O}$ uso de cateteres para aspiração de trombos, similar entre os grupos, não foi expressivo em nossa amostra e explica-se pelo fato de essa tecnologia ter sido incorporada em nossa rotina de atendimento apenas nos dois últimos anos do estudo.

A superioridade de resultados da intervenção coronária percutânea primária em relação à trombólise em idosos está bem demonstrada em estudos randomizados, sendo significativas as diferenças em relação aos desfechos de morte, reinfarto e acidente vascular cerebral, principalmente quando analisados de forma combinada. ${ }^{17,21}$ No entanto, em apresentação recente, 
Thomas Júnior NR, et al. Resultados Hospitalares da Intervenção Coronária Percutânea Primária no Infarto Agudo do Miocárdio em Pacientes com Mais de 80 Anos. Rev Bras Cardiol Invasiva. 2010;18(1):17-23.

o estudo SENIOR PAMI demonstrou que as diferenças entre esses desfechos eram mais significativas entre os idosos de 70 a 80 anos e bem menos evidentes nos pacientes com idade superior a 80 anos, caracterizando a complexidade desse grupo. ${ }^{8}$

A mortalidade hospitalar em nosso estudo, todos os casos de origem cardíaca, foi significativamente maior no grupo $\geq 80$ anos $(11,8 \%$ vs. $2,3 \%$; $P=0,014)$, com taxas semelhantes às da literatura. A análise conjunta dos estudos PAMI demonstrou mortalidade intrahospitalar de 10,2\% em pacientes $>75$ anos e de 1,8\% no grupo com idade $<75$ anos. $^{8}$ Esses estudos demonstraram, ainda, que, além da maior mortalidade, a intervenção coronária percutânea primária nos mais idosos apresenta risco aumentado de acidente vascular cerebral, sangramentos e insuficiência renal. Em nossa análise, no entanto, a ocorrência desses eventos não diferiu estatisticamente entre os grupos. Sabemos, porém, que na prática clínica esses eventos representam maior morbidade quando acometem os pacientes mais idosos.

\section{Limitações do estudo}

Reconhecemos como limitações deste estudo: 1) a análise retrospectiva dos dados entre duas coortes com variáveis clínicas não ajustadas; 2) o pequeno número de pacientes com mais de 80 anos de idade; 3) sua realização em um único centro; 4) a diversidade de operadores; e 5) a ausência de seguimento tardio.

\section{Recomendações}

Apesar de a literatura até o momento não demonstrar resultados muito superiores aos da trombólise, a intervenção coronária percutânea em pacientes muito idosos ( $\geq 80$ anos) não mostra maior número de complicações que possam contraindicar o método. Quando há disponibilidade de intervenção coronária percutânea, em nossa opinião, ela deve ser realizada, porém sempre com adequada utilização de dispositivos, fármacos e cuidados clínicos, quais sejam: stents, cateteres de aspiração de trombos, inibidores da glicoproteína IIb/IIla, balão de contrapulsação ou outro suporte de circulação assistida, e adequadas medidas de proteção renal e hemostasia do local da punção.

\section{CONCLUSÕES}

Este estudo demonstra, a partir de dados representativos do mundo real, que a intervenção coronária percutânea apresenta, em geral, altas taxas de sucesso angiográfico e baixo índice de complicações, reforçando seu papel como método preferencial de reperfusão na fase aguda do infarto agudo do miocárdio. Os pacientes muitos idosos ( $\geq 80$ anos) apresentam maior mortalidade, possivelmente reflexo da maior comorbidade desse grupo.

\section{CONFLITO DE INTERESSES}

Os autores declararam inexistência de conflito de interesses relacionado a este manuscrito.

\section{REFERÊNCIAS}

1. Simons LA. Epidemiologic considerations in cardiovascular diseases in the elderly: international comparisons and trends. Am J Cardiol. 1989;63(16):5H-8H.

2. Cardoso CO, Cardoso CR, Rodrigues LH, Staudt C, Duarte AF. Intervenção coronariana percutânea em pacientes com mais de 70 anos: análise de desfechos intra-hospitalares na era contemporânea. Rev Bras Cardiol Invasiva. 2008;16(1):64-9.

3. Kashani A, Giugliano RP. Management of ST-elevation myocardial infarction: an update on pharmacoinvasive recanalization. Am J Cardiovasc Drugs. 2008;8(3):187-97.

4. Berger PB, Ellis SG, Holmes DR, Granger CB, Criger DA. Relationship between delay in performing direct coronary angioplasty and early clinical outcome in patients with acute myocardial infarction: results from the Global Use of Strategies to Open Occluded Arteries in Acute Coronary Syndromes (GUSTO-IIb) Trial. Circulation. 1999;100(1):14-20.

5. Andersen HR, Nielsen TT, Rasmussen K, Thuesen L, Kelbaek $H$, Thayssen P. A comparison of coronary angioplasty with fibrinolytic therapy in acute myocardial infarction. $N$ Engl J Med. 2003;349(8):733-42.

6. Buchan I, Hooper L, McElduff P, Freemantle N, Martin D. Primary coronary angioplasty versus thrombolysis for acute myocardial infarction. Lancet. 2003;361(9365):1304.

7. DeBoer MJ, Ottervanger JP, Hof AW, Hoorntje JC, Suryapranata $\mathrm{H}$. Reperfusion therapy in elderly patients with acute myocardial infarction. J Am Coll Cardiol. 2002;39(11):1729-32.

8. Grines C. Senior PAMI: a prospective randomized trial of primary angioplasty and thrombolytic therapy in elderly patients with acute myocardial infarction. TCT 2005; October 16-21, 2005; Washington, DC.

9. Smith SC Jr, Feldman TE, Hirshfeld JW Jr, Jacobs AK, Kern MJ, King SB 3 ${ }^{\text {rd }}$, et al. ACC/AHA/SCAI 2005 guideline update for percutaneous coronary intervention: a report of the American College of Cardiology/American Heart Association Task Force on Practice Guidelines (ACC/AHA/SCAI Writing Committee to Update 2001 Guidelines for Percutaneous Coronary Intervention). Circulation. 2006;113(7):e166-286.

10. The Thrombolysis in Myocardial Infarction (TIMI) trial. Phase I findings. TIMI Study Group. N Engl J Med. 1985;312(14): 932-6.

11. Mattos LA, Lemos Neto PA, Rassi A Jr, Marin-Neto JA, Sousa AGMR, Devito FS, et al. Diretrizes da Sociedade Brasileira de Cardiologia. Intervenção coronária percutânea e métodos adjuntos diagnósticos em cardiologia intervencionista (II Edição - 2008). Rev Bras Cardiol Invasiva. 2008; 16 Supl. 2:9-88.

12. Kushner FG, Hand M, Smith SCJ, King SB, Anderson JL, Antman EM. 2009 focused updates: ACC/AHA Guidelines for the Management of Patients With ST-Elevation Myocardial Infarction (Updating the 2004 Guideline and 2007 Focused Update) and ACC/AHA/SCAI Guidelines on Percutaneous Coronary Intervention (Updating the 2005 Guideline and 2007 Focused Update). Circulation. 2009;120(22): 2271-306.

13. Alexander KP, Newby LK, Cannon CP, Armstrong PW, Gibler WB. Acute coronary care in the elderly, Part I. Non-STsegment-elevation acute coronary syndromes: a scientific statement for healthcare professionals from the American Heart Association Council on Clinical Cardiology: in collaboration 
Thomas Júnior NR, et al. Resultados Hospitalares da Intervenção Coronária Percutânea Primária no Infarto Agudo do Miocárdio em Pacientes com Mais de 80 Anos. Rev Bras Cardiol Invasiva. 2010;18(1):17-23.

with the Society of Geriatric Cardiology. Circulation. 2007; 115(19):2549-69.

14. Sousa AGMR, Costa JRJ, Abizaid A, Buitrón F, Costantini C, Marchant E. Perfil demográfico e resultados imediatos dos pacientes submetidos a angioplastia primária no registro SOLACI. Rev Bras Cardiol Invasiva. 2009;17(3):335-9.

15. Claessen BE, Kikkert WJ, Engstrom AE, Hoebers LP, Damman P. Primary percutaneous coronary intervention for ST elevation myocardial infarction in octagenarians; trends and outcomes. Heart. 2009 Dec 4. [Epub ahead of print]

16. Tavares Filho SC, Tanajura LF, Feres F, Centemero MP, Chaves AJ. Intervenção coronária percutânea em octogenários: houve mudanças no perfil dos casos tratados nos últimos cinco anos? Rev Bras Cardiol Invasiva. 2009;17(1):76-81.

17. DeGeare VS, Stone GW, Brodie BR, Cox DA, Garcia E. Angiographic and clinical characteristics associated with increased in-hospital mortality in elderly patients with acute myocardial infarction undergoing percutaneous intervention (a pooled analysis of the primary angioplasty in myocardial infarction trials). Am J Cardiol. 2000;86(1):30-4.

18. Lemos PA, Campos CA, Ribeiro EE, Falcão JL, Perin MA, et al. Incidência e preditores de óbito precoce e tardio em octagenários brasileiros tratados com intervenção coronária percutânea. Rev Bras Cardiol Invasiva. 2007;15(1):31-4.

19. Merchant FM, Weiner RB, Rao SR, Lawrence R, Healy JL. Inhospital outcomes of emergent and elective percutaneous coronary intervention in octogenarians. Coron Artery Dis. 2009;20(2):118-23.

20. Yan BP, Gurvitch R, Duffy SJ, Clark DJ, Sebastian M. An evaluation of octogenarians undergoing percutaneous coronary intervention from the Melbourne Intervention Group registry. Catheter Cardiovasc Interv. 2007;70(7):928-36.

21. Zhang Q, Zhang JS, Hu J, Yang ZK, Zheng AF. Outcomes of primary percutaneous coronary intervention for acute ST-elevation myocardial infarction in patients aged over 75 years. Ching Med. 2006;119(14):1151-6. 